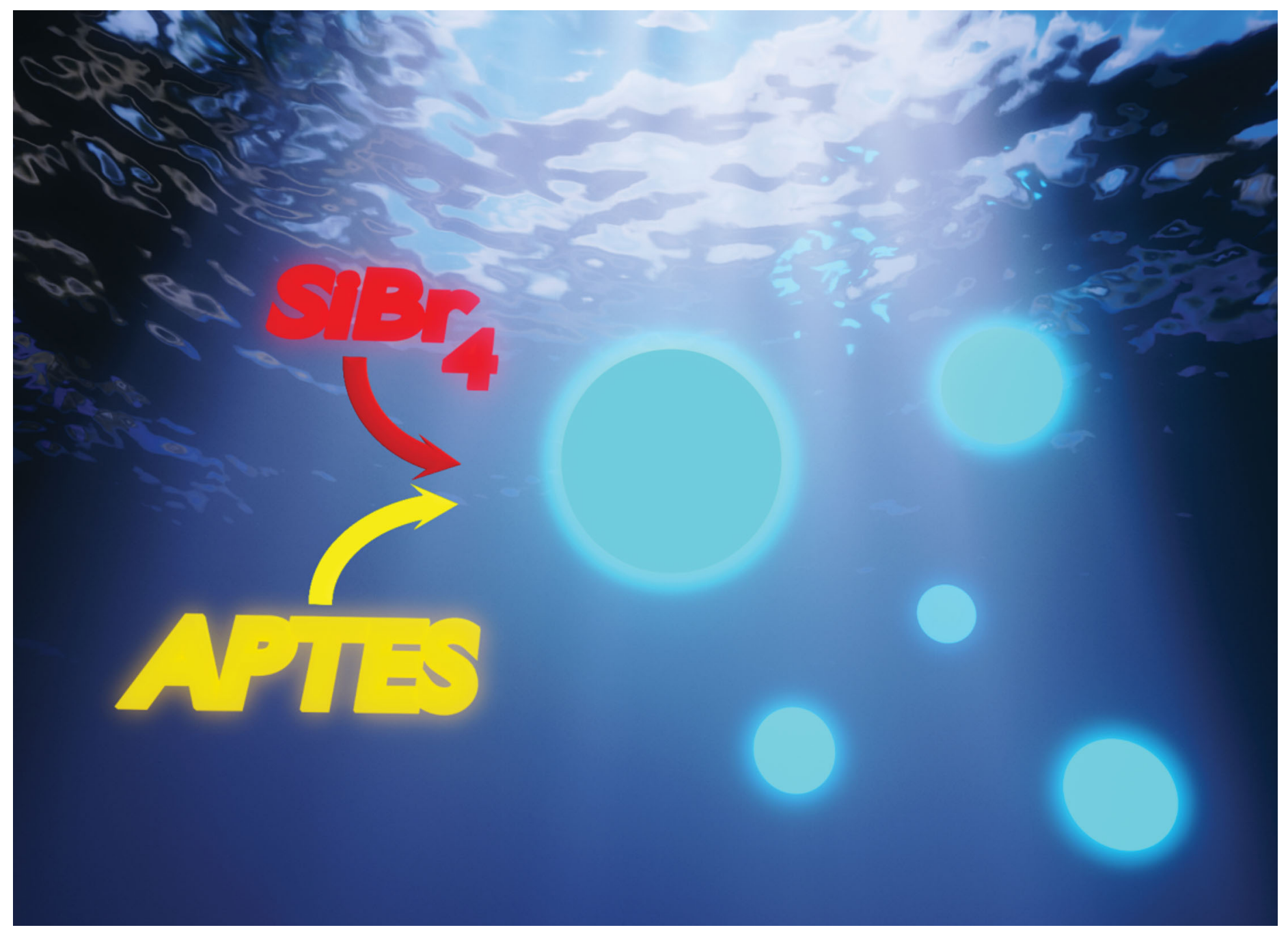

Highlighting research from the groups of Professor Françoise M. Winnik* and Dr Naoto Shirahata in the WPI International Center for Materials Nanoarchitectonics (MANA), National Institute for Materials Science (NIMS), Tsukuba, Japan (*also affiliated to the Department of Chemistry of the University of Montreal, Canada and the Department of Chemistry and Faculty of Pharmacy of the University of Helsinki, Helsinki, Finland).

A one-pot synthesis of water soluble highly fluorescent silica nanoparticles

Reaction of silicon tetrabromide with aminopropyltriethoxy silane yields photostable water-dispersible silica nanoparticles that emit blue light with an absolute quantum yield of $34 \%$.

\section{As featured in:}

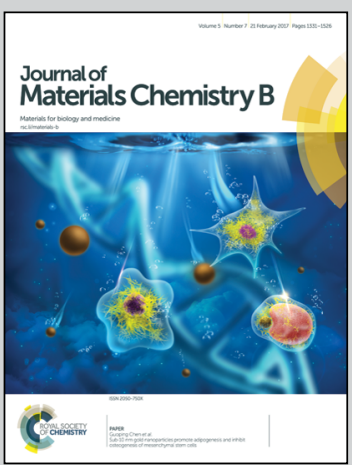

See Naoto Shirahata,

Françoise M. Winnik et al.,

J. Mater. Chem. B, 2017, 5, 1363. 


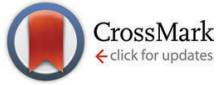

Cite this: J. Mater. Chem. B, 2017, 5, 1363

Received 27th October 2016, Accepted 13th December 2016

DOI: $10.1039 / c 6 t b 02813 f$

www.rsc.org/MaterialsB

\title{
A one-pot synthesis of water soluble highly fluorescent silica nanoparticles $\dagger$
}

\begin{abstract}
Sourov Chandra, ${ }^{a}$ Grégory Beaune, ${ }^{a}$ Naoto Shirahata*a ${ }^{\star a} d$ Françoise M. Winnik ${ }^{\star a b c}$
We report a one-pot synthesis of water dispersible fluorescent silica nanoparticles (NPs) functionalized with terminal amine groups, starting from silicon tetrabromide $\left(\mathrm{SiBr}_{4}\right)$ and aminopropyltriethoxy silane (APTES). The NPs range from 1 to $2 \mathrm{~nm}$ in diameter, and exhibit an intense blue emission with a quantum yield (QY) of around 34\% in water. They were characterized using XRD, XPS, TEM and FTIR spectroscopy for structural analysis. A tentative mechanism explaining the origin of the NPs emission in the blue region is presented based on the distinctive features of their low temperature photoluminescence $(\mathrm{PL})$, photoluminescence excitation (PLE) spectrum and time correlated single photon counting lifetime decay profiles. The outstanding PL QY and photostability of the NPs, together with their water dispersibility and biocompatibility, constitute a unique set of properties among existing silica NPs and enable the application of the NPs in various fields.
\end{abstract}

\section{Introduction}

Photoluminescent functionalized silica nanoparticles (NPs) are promising materials for biomedical applications, especially in optical imaging and drug delivery in view of their high photoluminescence quantum yields (PL QYs), outstanding photostability, colloidal stability and biocompatibility. ${ }^{1-4}$ Surface functionalization introduces an additional feature, providing opportunities to connect antibodies, polymers, dyes, and receptors to the silica surface. ${ }^{5}$ There are several methods available for the synthesis of silica NPs, in which the Stober method is the most commonly used. In this method, monodisperse silica spheres are grown via the controlled hydrolysis and condensation of tetraethyl orthosilicate (TEOS) in the presence of ammonia as a catalyst. ${ }^{6}$ The particles obtained in alkaline media are non-luminescent in nature. Organic or inorganic fluorophores can be entrapped within the silica matrix or framework via noncovalent interactions. ${ }^{7-9}$ The dye-loaded silica NPs are less toxic and more resistant against photobleaching compared to the corresponding free dyes. ${ }^{10}$ In addition, the outer surface of the dye-loaded NPs can be used for further functionalization. ${ }^{11}$ Dyed silica NPs emitting throughout the UV-visible to the NIR region are currently available, but their high tendency to release

\footnotetext{
${ }^{a}$ WPI International Centre for Nanoarchitectonics (MANA), National Institute for Materials Science (NIMS), 1-1 Namiki, Tsukuba 305-0044, Japan. E-mail: SHIRAHATA.Naoto@nims.go.jp

${ }^{b}$ Departement de Chimie, Universite de Montreal, CP 6128 Succursale Centre Ville, Montreal QC H3C 3J7, Canada. E-mail: francoise.winnik@umontreal.ca 'Department of Chemistry and Faculty of Pharmacy, University of Helsinki, FIN 00014 Helsinki, Finland

† Electronic supplementary information (ESI) available. See DOI: 10.1039/c6tb02813f
}

some of the entrapped fluorophores and photobleaching prevent their use in vivo for long term applications. ${ }^{12}$ Hybrid inorganic materials, such as quantum dot- ${ }^{7}$ or lanthanide- ${ }^{13}$ loaded silica NPs, have been used as photostable alternatives to dyed NPs. However, their practical applications, particularly in vivo, remain limited in view of their tedious, multistep synthesis and concerns related to their toxicity. ${ }^{14}$

Jakob et al. reported the synthesis of strongly emissive monodisperse silica spheres by calcination of aminopropyl silica obtained by co-mixing of TEOS and aminopropyltriethoxy silane (APTES) in an ammonia/water/ethanol solution. ${ }^{15}$ The optical properties of the resulting silica spheres vary, depending on the calcination temperature and the APTES to TEOS molar ratio. The highest PL QY (12\%) with an emission maximum at $430 \mathrm{~nm}$ was achieved when a fraction of APTES was kept low $(3 \%)$ and the calcination temperature was set at $400{ }^{\circ} \mathrm{C}$. Interestingly, silica spheres prepared without APTES were almost non-luminescent. Later, Brites et al. reported a low temperature $\left(45{ }^{\circ} \mathrm{C}\right)$ preparation of organosilica NPs using a modified Stober method using TEOS and APTES as the precursor. ${ }^{16}$ The resulting NPs emitted light between 430 and $500 \mathrm{~nm}$ with PL QY up to $15 \%$. White light emitting silica nanostructures were obtained via acid catalyzed sol gel reaction of TEOS. ${ }^{17}$ In addition, Mahtab et al. reported the synthesis of magnetic silica NPs with green PL emission and a PL QY of around 7.1\%. ${ }^{18}$ Here, we report a new synthesis of amine-functionalized silica NPs 1 to $2 \mathrm{~nm}$ in diameter via a solvothermal reaction between $\mathrm{SiBr}_{4}$ and APTES at $200{ }^{\circ} \mathrm{C}$. The resulting NPs disperse readily in water, yielding colloidally stable dispersions that resist aggregation and photobleaching upon storage under bright ambient conditions for several months. The NPs emit an intense blue light with a PL 
QY around 34\%, the highest QY value reported so far for silica NPs. These highly efficient, non-photobleaching, biocompatible blue-luminescent NPs outperform commercially available products used in light harvesting applications and pharmacokinetic measurements and, in view of their simple synthesis, could eventually replace them.

\section{Materials and method}

\subsection{Materials}

Water was purified and deionized using a Sartorius (Arium 611 UV) water purification system. Aminopropyltriethoxysilane (APTES, 99\%) and silicon tetrabromide ( $\left.\mathrm{SiBr}_{4}, 99.995 \%\right)$ were obtained from Sigma Aldrich and used as received. All other chemicals were purchased from Wako pure chemical industries Ltd (Japan) and used as received. Trypan blue and Dulbecco's modified Eagle's medium (DMEM) were purchased from Gibco. The CCK-8 kit was received from Dojindo Molecular Technologies (Japan). NIH3T3 and Hek 293 cell lines were collected from the Riken Cell Bank (Japan). Fetal bovine serum (FBS) was purchased from Sigma Aldrich. Trypsin-EDTA (0.05\%) and penicillinstreptomycin were purchased from Life Technologies.

\subsection{Synthesis of silica NPs}

A stirred mixture of ethylene glycol $(10 \mathrm{~mL})$ and water $(5 \mathrm{~mL})$, kept under a flow of argon at $25{ }^{\circ} \mathrm{C}$ was treated sequentially with $\mathrm{SiBr}_{4}(100 \mu \mathrm{L}, 0.805 \mathrm{mmol})$ and APTES (3 mL, $\left.0.128 \mathrm{~mol}\right)$. The solution was stirred for 30 minutes. The resulting milky suspension was transferred in a $30 \mathrm{~mL}$-Teflon container. The sealed container was inserted in a stainless steel autoclave. The autoclave was brought to $200{ }^{\circ} \mathrm{C}$ with a heating rate of $4{ }^{\circ} \mathrm{C} \min ^{-1}$, kept at this temperature for $15 \mathrm{~h}$ and brought back to room temperature. The resultant yellowish-green solution was dispersed in water. The product was purified by dialysis (Spectra/Por Dialysis Membrane, MWCO: 3500) in deionized water for $15 \mathrm{~h}$ to remove ethylene glycol and other impurities. The NPs precipitated during dialysis. They were collected by centrifugation (15000 rpm, $20 \mathrm{~min}$ ), washed with water $(100 \mathrm{~mL})$ several times, and dried under vacuum. The powder was insoluble in neutral water but was readily soluble in aqueous $\mathrm{NaOH}(1 \mathrm{~N})$, yielding a solution having the same UV-visible absorption spectrum as the crude product, but a significantly reduced PL. Control syntheses were performed under the same protocol using either $\operatorname{SiBr}_{4}(100 \mu \mathrm{L}, 0.805 \mathrm{mmol})$ alone or APTES ( $3 \mathrm{~mL}$ and $100 \mu \mathrm{L}$ ) alone dissolved in $10 \mathrm{~mL}$ of ethylene glycol in $5 \mathrm{~mL}$ of water and heated at $200{ }^{\circ} \mathrm{C}$ for $15 \mathrm{~h}$, all other conditions being kept unaltered.

\subsection{Characterization}

Fourier Transform Infrared (FTIR) spectroscopy measurements were carried out on a JASCO FT/IR 4100 spectrometer, using powder samples placed in the sample holder. UV-visible absorption spectra were recorded on a JASCO V-650 spectrophotometer. $\mathrm{X}$-ray photoelectron spectroscopy (XPS) was performed using a Shimadzu, ESCA3400 using $\operatorname{MgK}_{\alpha}(E=1253.6 \mathrm{eV})$ radiation.
The binding energy (BE) scale was calibrated to provide $\mathrm{Pt}_{4} \mathrm{f}_{7 / 2}=71.1 \mathrm{eV}$. The X-ray source was operated at $10 \mathrm{~mA}$ and $12 \mathrm{kV}$. The core-level signals were obtained with a photoelectron take-off angle of $90^{\circ}$ with respect to the sample surface. Data acquisition and processing were performed by a SUN Microsystems ULTRA 5 computer, using the VISION 2.0 processing package. The accuracy of the BE determined with respect to the standard value was within $\pm 0.3 \mathrm{eV}$. X-ray diffraction (XRD) was carried out with a Rigaku Smart lab X-ray diffractometer, operated in ambient air. Dynamic light scattering (DLS) and zeta potential measurements were performed on aqueous NP dispersions kept at $25{ }^{\circ} \mathrm{C}$ using an ELSZ-2000 zeta-potential and particle size analyzer (Otsuka Electronics Co., Ltd). High resolution transmission electron microscopy (HR-TEM) and selected area electron diffraction (SAED) pattern measurements were performed with a JEOL JEM 2010, operated at an acceleration voltage of $200 \mathrm{kV}$. Samples for HR-TEM analysis were drop-cast on a copper grid from a very dilute aqueous particle suspension. PL and PLE spectra of aqueous silica NP dispersions were recorded using a NanoLog Horiba Jovin Yvon spectrofluorometer. Time-resolved fluorescence decay profiles were obtained with a time-correlated single photon counting (TCSPC) lifetime spectroscopy system (NanoLog, Horiba Jovin Ybon, Japan), equipped with pulse laser diodes $\left(\lambda_{\mathrm{em}}=370 \mathrm{~nm}\right)$. The quality of the fit was assessed based on the $\chi^{2}$ value $(\sim 1.0)$ and visual inspection of the residuals. Absolute PL QYs were estimated by the standardized integrating-sphere method using a Hamamatsu C9920-02 Photonics system, equipped with a $150 \mathrm{~W}$ xenon lamp. Dilute aqueous solutions with an absorbance in between 0.1 and 0.2 at $304 \mathrm{~nm}$ wavelength were taken in $10 \mathrm{~mm}^{2}$ quartz cuvettes and were placed into the instrument sample compartment.

\subsection{Cytotoxicity assays}

For cellular assays, silica NP suspensions were prepared by dilution of the particles in water $(\mathrm{pH}=7.0)$.

Trypan blue test. Fibroblasts non-cancerous murine cells (NIH3T3) were seeded in a 24-wells plate containing the DMEM medium supplemented with $10 \%$ fetal bovine serum (FBS), 100 units per $\mathrm{mL}$ penicillin, and $100 \mathrm{mg} \mathrm{mL}^{-1}$ streptomycin at $37{ }^{\circ} \mathrm{C}$ in humidified air contains $5 \% \mathrm{CO}_{2}$. After 1 day, the cells were treated with increasing concentrations of silica NP $\left(25,50,100,200,300\right.$ and $\left.500 \mu \mathrm{g} \mathrm{mL}^{-1}\right)$ and further incubated for $24 \mathrm{~h}$. The cells were washed thrice with PBS and trypsinized. Trypan blue $(0.4 \% \mathrm{wt} / \mathrm{vol}, 100 \mu \mathrm{L})$ was added to an aliquot of the cell suspension $(100 \mu \mathrm{L})$ and incubated for $5 \mathrm{~min}$ at room temperature. The viable cells were counted by bright field microscopy. The viability values of the silica NP treated cells are expressed as a percentage of the viability of control cells. All experiments were repeated 3 times.

CCK-8 assay. The viability of the NIH3T3 cells was checked using a CCK-8 kit (Dojindo Molecular Technologies, Japan) according to the manufacturer's instructions. Briefly, a NIH3T3 cell suspension $(100 \mu \mathrm{L}, 5000$ cells per well) was dispersed in a 96-well plate containing DMEM medium supplemented with $10 \%$ fetal bovine serum (FBS), 100 units per $\mathrm{mL}$ penicillin, and $100 \mathrm{mg} \mathrm{mL} \mathrm{m}^{-1}$ streptomycin. The plate was pre-incubated at 
$37{ }^{\circ} \mathrm{C}$ in humidified air containing $5 \% \mathrm{CO}_{2}$ for 1 day. Then, silica NP suspensions $(10 \mu \mathrm{L})$ were added into the wells to final concentrations of $25,50,100,200,300$, and $500 \mu \mathrm{g} \mathrm{mL}^{-1}$. The plate was incubated for $24 \mathrm{~h}$ under the same conditions mentioned above. A CCK-8 solution $(10 \mu \mathrm{L})$ was added to each well and the plate was incubated for $4 \mathrm{~h}$ in the incubator. Finally, the absorbance at $450 \mathrm{~nm}$ of each well was measured using an MTP-800 microplate reader (Corona Electric).

\subsection{Cellular uptake and confocal microscopy}

NIH3T3 cells were plated on glass cover slips at a concentration of $10^{5}$ cells per $\mathrm{mL}$. They were treated with the particle suspension in water $\left(100 \mu \mathrm{g} \mathrm{mL}^{-1}\right)$ and incubated for $24 \mathrm{~h}$. They were washed thrice with PBS buffer (5 minute each time) and fixed with paraformaldehyde. Cells were imaged on a confocal microscope (TCS-SP5 confocal microscope, Leica Microsystems, $\left.\lambda_{\mathrm{ex}}=405 \mathrm{~nm}\right)$.

\section{Results and discussion}

\subsection{Synthesis and characterization of Silica NPs}

We report a solvothermal approach to obtain amorphous silica NPs through the reaction between $\mathrm{SiBr}_{4}$ and APTES in water as depicted schematically in Fig. 1A. It is well recognized that $\mathrm{SiBr}_{4}$ is readily hydrolyzed in contact with water to form $\mathrm{Si}(\mathrm{OH})_{4}$, eventually converted into silicon oxide. APTES has a strong tendency to form a covalently-bound monolayer on chemically grown silicon oxide. ${ }^{19,20}$ Thus, in this synthesis, $\mathrm{SiBr}_{4}$ immediately hydrolyzes to $\mathrm{Si}(\mathrm{OH})_{4}$ in the presence of an ethylene glycol (EG)/water mixture and, when kept under pressure at high temperature, it polymerizes to form -(Si-O $)_{x}$ stabilized in water by hydrolyzed APTES bound to the surface. The APTES grafting stops the growth of $-(\mathrm{Si}-\mathrm{O})_{x}$ and introduces amine groups tethered to the $-(\mathrm{Si}-\mathrm{O})_{x}$ surface. The resulting colloidal NPs emit an intense blue luminescence under UV irradiation. Their aqueous dispersions resist precipitation even after centrifugation at $100000 \mathrm{rpm}$.

The validity of the mechanism proposed is strengthened by our observations that the same procedure performed with either only $\mathrm{SiBr}_{4}$ or only APTES fails to produce emissive silica NPs, yielding exclusively non-emissive macroscopic aggregates. Fig. 1B presents photographs of aqueous dispersions of the products in water obtained using APTES and $\mathrm{SiBr}_{4}$ together or separately under the same conditions. Silica NPs form only when the reaction is conducted with both APTES and $\mathrm{SiBr}_{4}$ in a water/EG system. The resulting sample (vial a) is a clear liquid with a yellowish-green hue. However, when the reaction is executed with $\mathrm{SiBr}_{4}$ alone, a colorless liquid is formed together with some yellow precipitate (vial b). Using APTES alone, the process leads to the formation of a yellow liquid and a brownish floc, irrespective of the starting amount of APTES (vials $\mathrm{c}$ and $\mathrm{d}$ ). Fig. 1C demonstrates that the silica NPs emit bright blue luminescence under UV irradiation.

The silica nanoparticles were analyzed using FTIR spectroscopy and XPS in order to ascertain their composition and surface chemistry (Fig. 2). The FTIR spectrum shown in Fig. 2a presents a strong band at $1040 \mathrm{~cm}^{-1}$ characteristic of the $\mathrm{Si}-\mathrm{O}$ linkage as well as a band at $1552 \mathrm{~cm}^{-1}$, typical of the
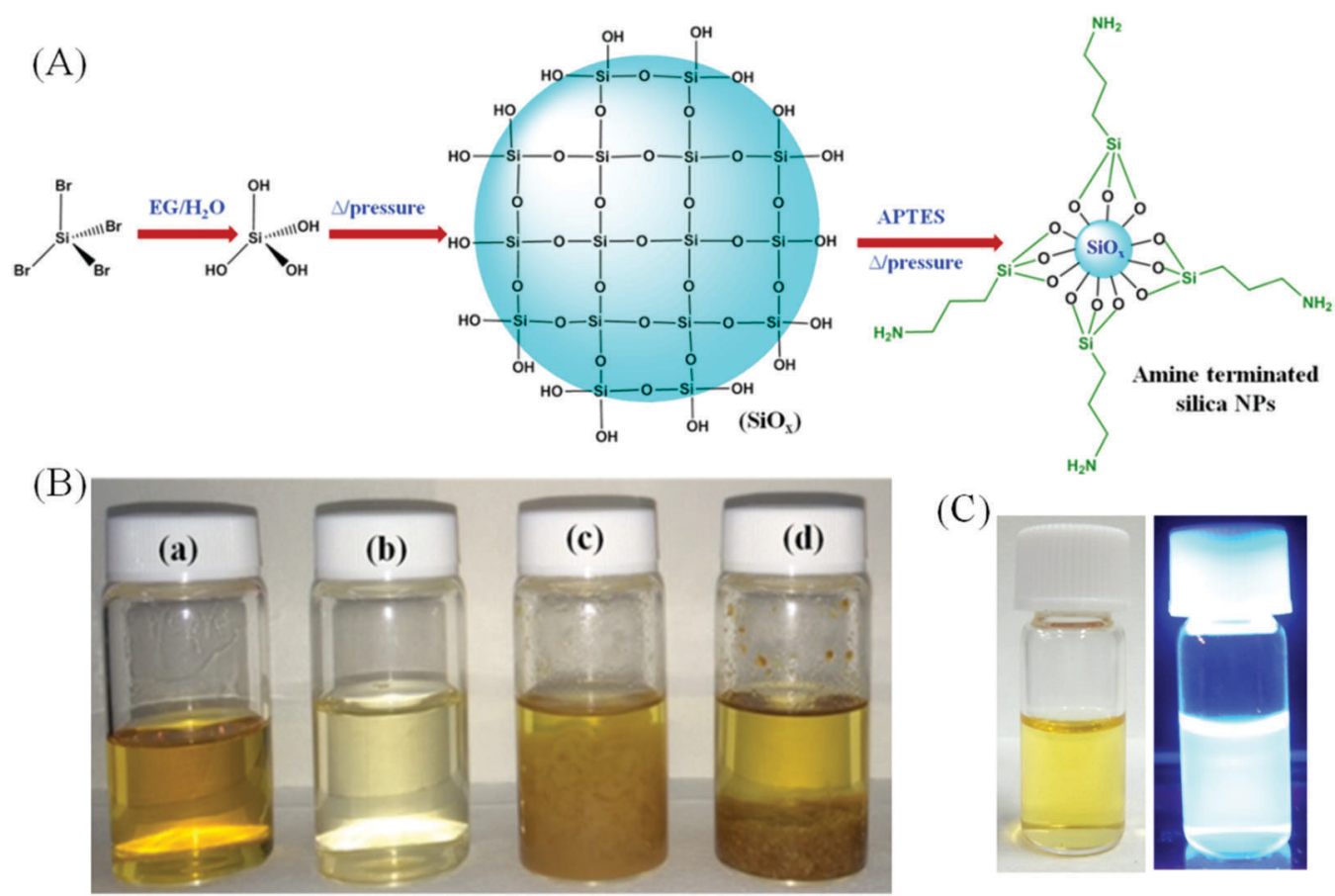

Fig. 1 (A) Schematic representation for the formation of silica NPs. (B) Photographs of the products, prepared by using (a) SiBr ${ }_{4}$ and APTES in the $\mathrm{EG} /$ water system, (b) $\mathrm{SiBr}_{4}$ alone in the $\mathrm{EG} /$ water system, (c) APTES alone $(100 \mu \mathrm{L})$ in the $\mathrm{EG} /$ water system and (d) APTES alone (3 $\left.\mathrm{mL}\right)$ in the EG/water system. (C) Photographs of silica NPs in water under (a) white light and (b) UV light. 

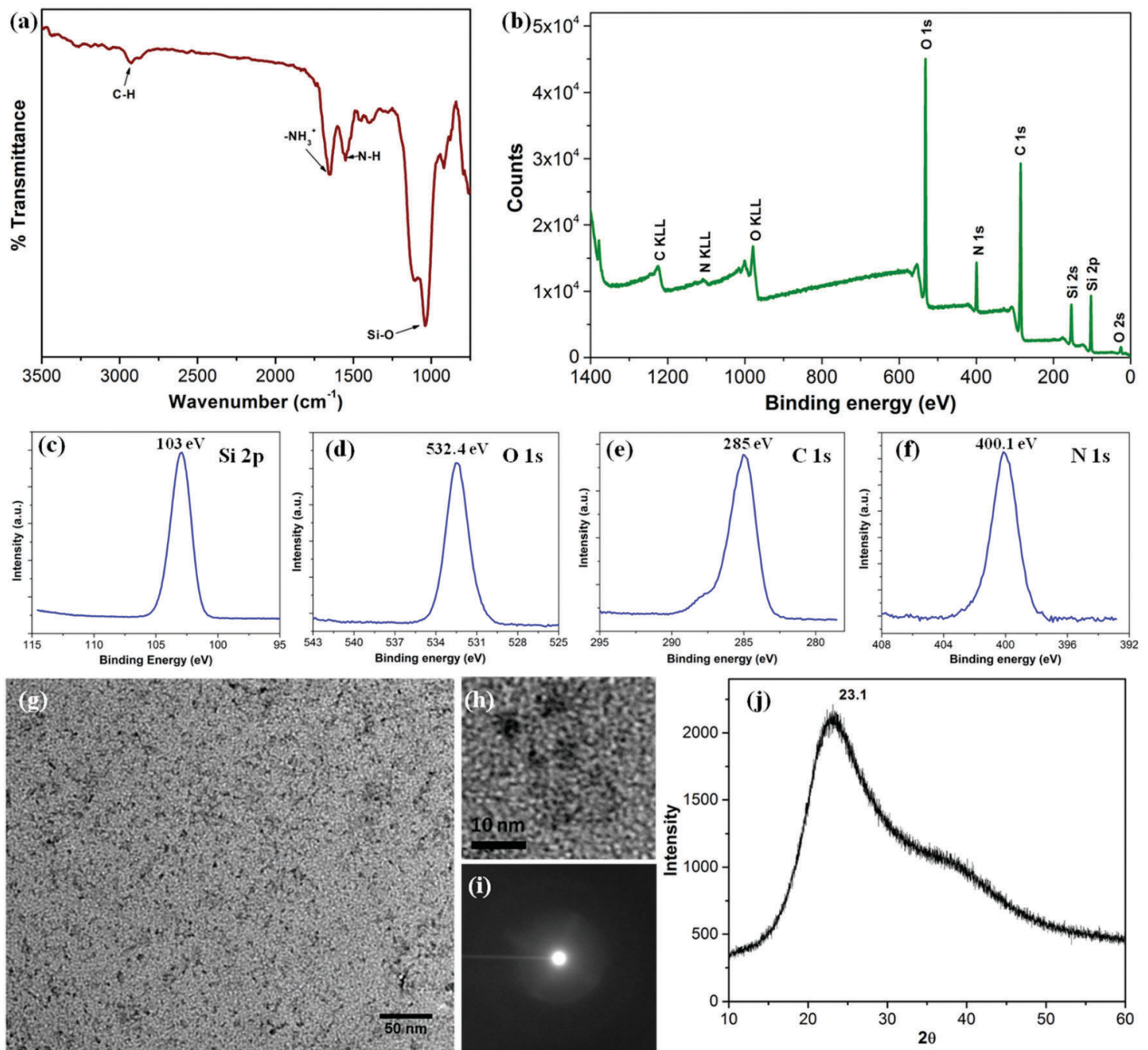

Fig. 2 (a) FTIR spectrum and (b) wide scan XPS spectrum of the silica NPs; the enlarged regions of the XPS spectrum of Si2p (c), O1s (d), C1s (e) and N1s (f). (g) TEM image, (h) HR-TEM image, (i) SAED pattern and (j) X-ray diffraction pattern of the silica NPs.

amine vibrations. The presence of both bands is consistent with the mechanism proposed above, which involves first the formation of $-(\mathrm{Si}-\mathrm{O})_{x}$ from $\mathrm{SiBr}_{4}$ followed by the reaction of $-(\mathrm{Si}-\mathrm{O})_{x}$ with hydrolyzed APTES. ${ }^{21}$ The sharp band at $1655 \mathrm{~cm}^{-1}$ is assigned to the asymmetric $-\mathrm{NH}_{3}{ }^{+}$deformation mode. The water molecules retained by siliceous materials could also account for this band. A small band at $2930 \mathrm{~cm}^{-1}$, assigned to $\mathrm{C}-\mathrm{H}$ bond vibrations provides further evidence for the presence of APTES moieties. ${ }^{19,21}$ The presence of functional groups on the outer surface of the silica NPs and the mode of bonding in between the elements was confirmed by analysis of the XPS spectra shown in Fig. 2b. The wide XPS scan displays signals due to carbon (53.2\%), oxygen (28.1\%), silicon (10.6\%) and nitrogen $(8.1 \%)$. The prominent peaks for Si2s, Si2p, O1s, C1s and N1s are consistent with the formation of silica NPs bearing hydrolyzed APTES molecules on their surface. The Si2p spectrum (Fig. 2c) consists of a distinct peak centered at $103 \mathrm{eV}$, characteristic of Si-O bonds. ${ }^{22}$ The C1s peak (Fig. 2e) centered at $285 \mathrm{eV}$ supports the existence of the $\mathrm{C}-\mathrm{C}$ bonds of the hydrolyzed APTES moieties. Finally, the N1s spectrum (Fig. 2f) at $400.1 \mathrm{eV}$ confirms the presence of primary amine $\mathrm{N}-\mathrm{H}$ bonds. ${ }^{23}$ Fig. $2 \mathrm{~g}$ and $\mathrm{h}$ present low and high resolution TEM micrographs of the silica NPs, which are spherical in shape with diameters of 1-2 $\mathrm{nm}$. The NPs are very small in size. They are uniformly distributed without signs of agglomeration. The corresponding SAED pattern (Fig. 2i) reveals that they are amorphous in nature, as confirmed by the X-ray diffraction pattern shown in Fig. $2 \mathrm{j}$ which presents a broad featureless signal centered at $2 \theta=23.1$. The average NP size calculated from the diffraction peak is around $1.2 \mathrm{~nm}$, very close to the result obtains from TEM image. The hydrodynamic diameter of the NPs in water, determined by DLS, was $1.2 \pm 0.2 \mathrm{~nm}$, in excellent agreement with the TEM and XRD data (Fig. S1, ESI $\dagger$ ). The zeta potential values of the particles dispersed in aqueous media of $\mathrm{pH}<7$ were positive in agreement with the presence of surface $-\mathrm{NH}_{3}{ }^{+}$functionalities (Fig. S2, ESI $\dagger$ ). Low positive zeta potential with very high stability in an aqueous medium could be explained by the presence over the surface of the silica 
NPs, of tethered EG groups responsible for charge shielding and preventing their agglomeration in solution. ${ }^{24,25}$

\subsection{Optical properties}

The UV-Vis absorption spectrum of the as-synthesized bright greenish yellow aqueous silica NPs dispersion in water consists of two distinct bands: a strong band centered at $304 \mathrm{~nm}$ (major) and a weak band around $429 \mathrm{~nm}$ (Fig. 3a). This strong and broad absorption permits excitation over a long wavelength range ${ }^{26}$ as shown in Fig. $3 \mathrm{~b}$, which presents the emission spectra of the silica NPs in water using excitation wavelength from 300 to $360 \mathrm{~nm}$. The emission undergoes red shift with increasing excitation wavelength. This shift may be due to the presence of emissive traps associated with the oxygen or amine functionalities on the silica surface. ${ }^{27}$ The highest emission intensity, centered at $410 \mathrm{~nm}$, was recorded upon excitation at $310 \mathrm{~nm}$ (Fig. 3c). The estimated PL full width half maximum (FWHM) is about $105 \mathrm{~nm}$. The PL QY of this silica NP excited at $310 \mathrm{~nm}$ is approximately $34.3 \%$, which is the highest value reported so far for emissive silica NPs. The PL QY value is about $31.2 \%, 34 \%, 27.1 \%, 25.3 \%, 24.5 \%$ and $23.2 \%$ upon excitation at 300, 320, 330, 340, 350 and $360 \mathrm{~nm}$, respectively (Fig. S3, ESI $\dagger$ ). The PL intensity is not influenced by changes in the $\mathrm{pH}$ of the medium from 1 to 14 (Fig. S4, ESI $\dagger$ ). The PL QY of silica NP remained constant over the entire range. The emission underwent a small red shift for samples at $\mathrm{pH} 1$. The NPs are highly photostable. No photobleaching was detected upon UV irradiation (4 Watt, $365 \mathrm{~nm}$ ) over an $8 \mathrm{~h}$ period (Fig. S5, ESI $\dagger$ ). The Commission Internationale de I'Eclairage (CIE) diagram shown in Fig. 3d, reveals the CIE coordinates (1931) $x=0.15262, y=0.03345$, corresponding to the blue region of the visible light. The origin of this blue luminescence is still a matter of debate as discussed in the next section.

\subsection{Emission mechanism}

In order to determine the origin of this blue emission, we have carried out an extensive photophysical analysis that included PLE spectroscopy, low temperature PL emission spectroscopy, and TCSPC lifetime decay analysis. Fig. 4a displays the normalized PL and PLE spectra of the silica NP obtained, respectively, with an excitation wavelength of $310 \mathrm{~nm}$ and an emission wavelength of $410 \mathrm{~nm}$. The PL spectrum is centered at $410 \mathrm{~nm}$ and the PLE spectrum has a maximum at $308 \mathrm{~nm}$, confirming that the broad emission band corresponds to the PLE excitation energy. ${ }^{28,29}$ The highest PLE intensity has been achieved with a $410 \mathrm{~nm}$ emission. The PLE intensity gradually diminishes as the emission wavelength increases from 410 to $450 \mathrm{~nm}$ (Fig. 4b), indicating that the PL efficiency is a linear function of the PLE excitation. Interestingly, the PLE spectrum remains centered at
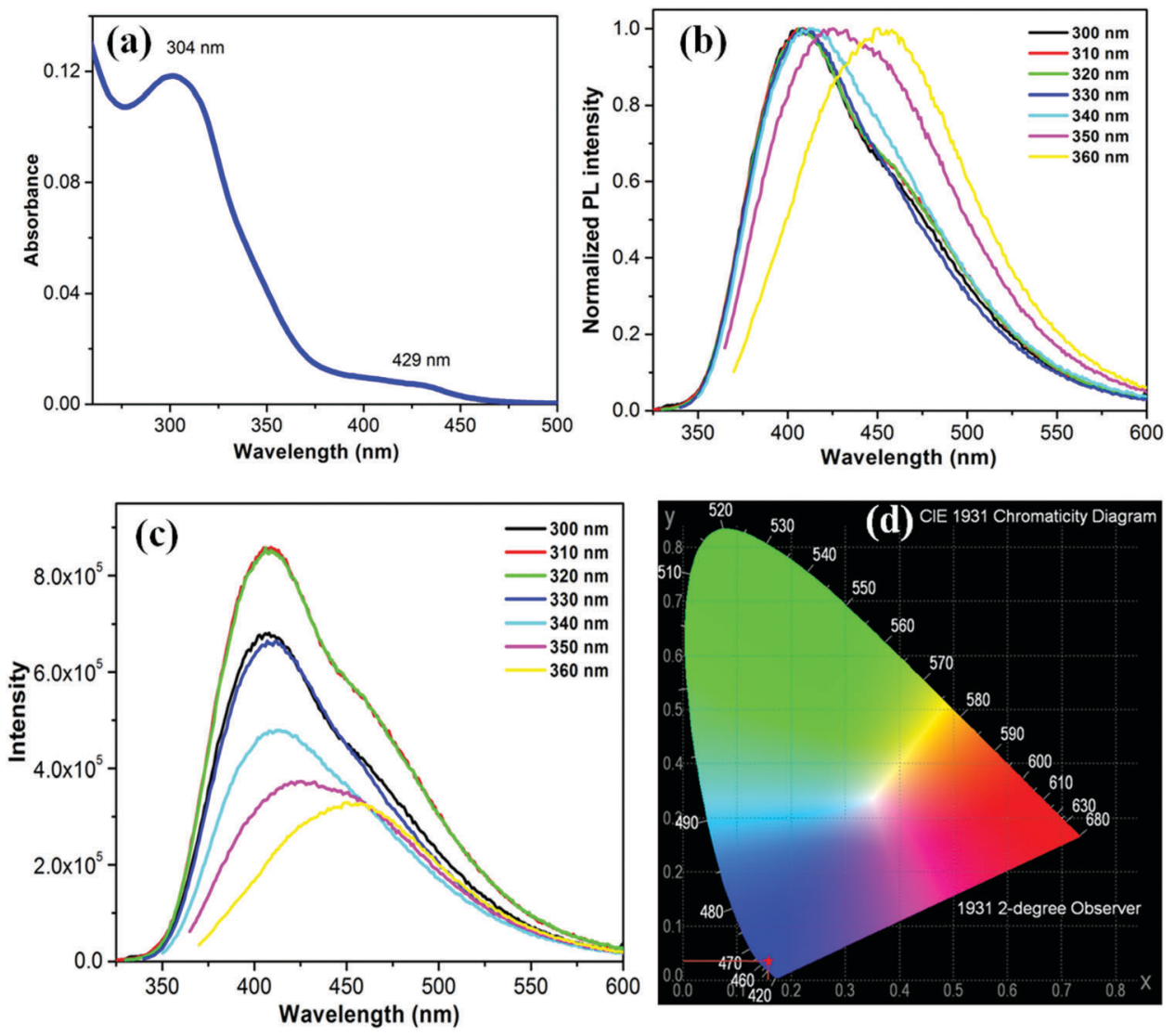

Fig. 3 (a) UV-visible spectrum, (b) normalized PL spectra at different excitation wavelength and (c) corresponding PL spectra of silica NP. (d) CIE diagram of silica NP, showing chromaticity coordinates of the sample. 

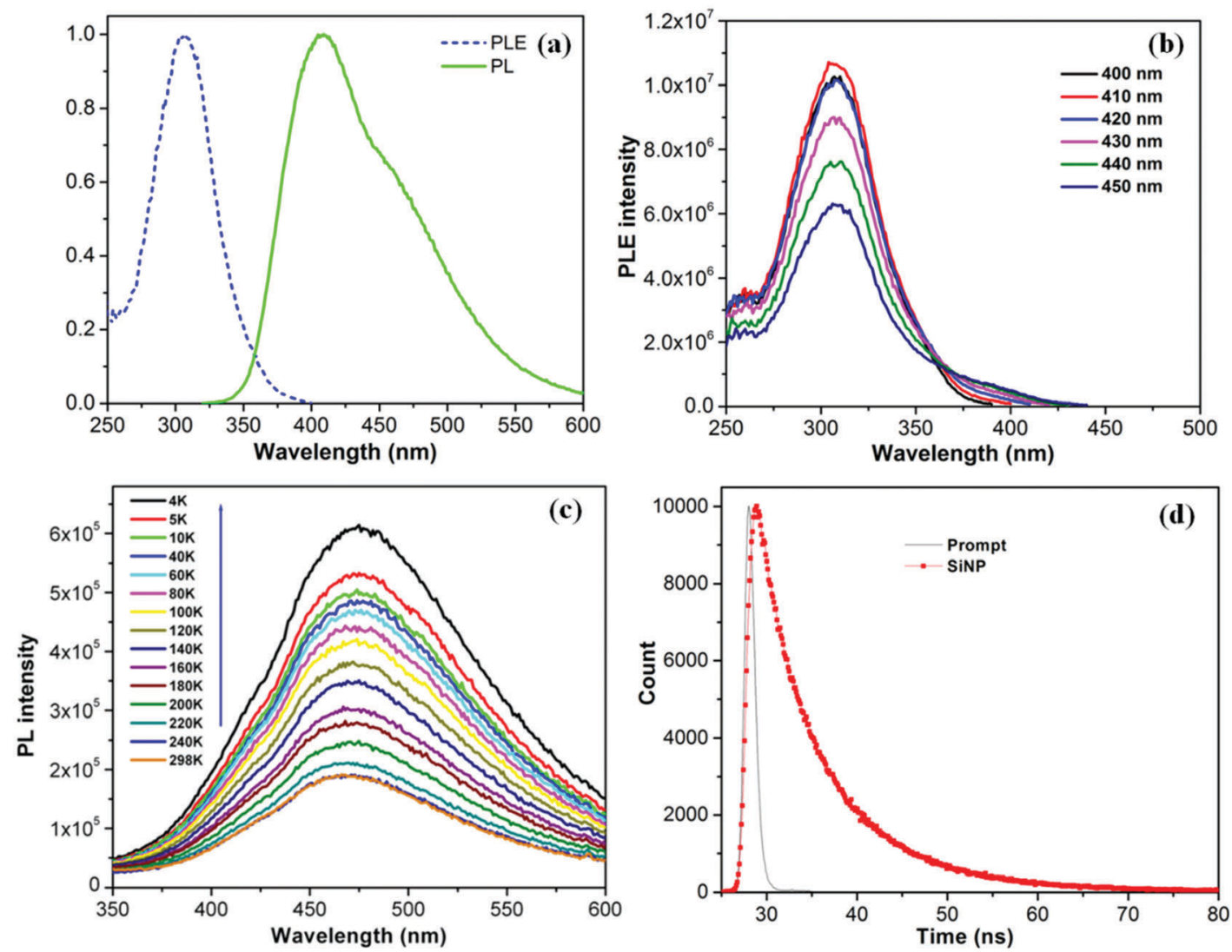

Fig. 4 (a) Normalized PLE and PL spectrum of silica NPs at $410 \mathrm{~nm}$ emission and $310 \mathrm{~nm}$ excitation respectively, (b) PLE spectrum of silica NPs at different emission wavelength. (c) Temperature dependent PL emission of silica NPs, deposited on a quartz surface. (d) TCSPC life time decay profile of the silica NPs.

$308 \mathrm{~nm}$ (Fig. S6, ESI $\dagger$ ), irrespective of the emission wavelength monitored. Moreover the PLE spectra coincide well with the UV-visible absorption spectra of the NPs. The PL intensity of solid NPs deposited on quartz decreases with increasing temperature from $4 \mathrm{~K}$ to $298 \mathrm{~K}$ (Fig. 4c). Unlike the large red shifts in energy gap observed for crystalline particles, ${ }^{30}$ silica NPs exhibit a slight blue shift of $34 \mathrm{meV}$ as the temperature passes from $4 \mathrm{~K}$ to $298 \mathrm{~K}$. This shift is attributed to the enhanced interaction of electron-phonon scattering with increasing temperature. ${ }^{31}$ The result suggests that electron-phonon interactions and electronsurface defect scattering play an important role in the PL properties of the very small sized silica NPs, as discussed by Kwack et $a l^{32}$ At high temperatures, several nonradiative channels are thermally activated due to the formation of surface trapping states caused by surface defects caused by impurities, such as carbon and oxygen atoms, and by the nitrogen centers on the surface. ${ }^{30,33,34}$ It has been reported that the structural disorder associated with the amorphous state enhances PL efficiency in amorphous particles. ${ }^{32,35}$ The PL lifetime decay profile of silica NP (Fig. 4d) was fitted to a bi-exponential function, with lifetimes $\tau_{1}=3.97 \mathrm{~ns}$ and $\tau_{2}=10.19 \mathrm{~ns}$; the average life time is $4.27 \mathrm{~ns}$. The local atomic distortions on the electronic structure due to the insertion of oxygen in amorphous silicon oxide are believed to be responsible for the high radiative recombination rate. ${ }^{36,37}$

\subsection{In vitro cytotoxicity and bio-imaging}

CCK-8 and trypan blue tests were performed on two cell lines, Hek-293 and NIH3T3, to assess the silica NPs cytotoxicity. ${ }^{38}$ The experimental results of both assays (Fig. $5 \mathrm{a}$ and $\mathrm{b}$ ) indicate that the NPs are non toxic up to the concentration of $500 \mu \mathrm{g} \mathrm{mL} \mathrm{m}^{-1}$ for the Hek293 and NIH3T3 cell lines. To ascertain the intracellular uptake of these NPs, fluorescence imaging of NPtreated cells were carried out on NIH3T3 mouse embryonic fibroblast cells (Fig. 6A). After a 24 h-incubation with $100 \mu \mathrm{L}$ $\left(100 \mu \mathrm{g} \mathrm{mL}^{-1}\right)$ of NPs, they are located in the cell cytoplasm and emit intense blue fluorescence light. Z-stack images in NIH3T3 cells shown in Fig. 6B confirm that the NPs are equally distributed over the interior of the cells. After insertion, the particles tend to accumulate at the edge of the nucleus but not inside.

\section{Conclusions}

We prepared amine functionalized water-dispersible silica NPs 1-2 nm in diameter. The NPs emit intense blue PL without photobleaching. An elevated rate of radiative recombination due to localized surface states present in amorphous silica is believed to be responsible for the NPs high PL intensity with QY > 34\%. The NPs are non-toxic. 

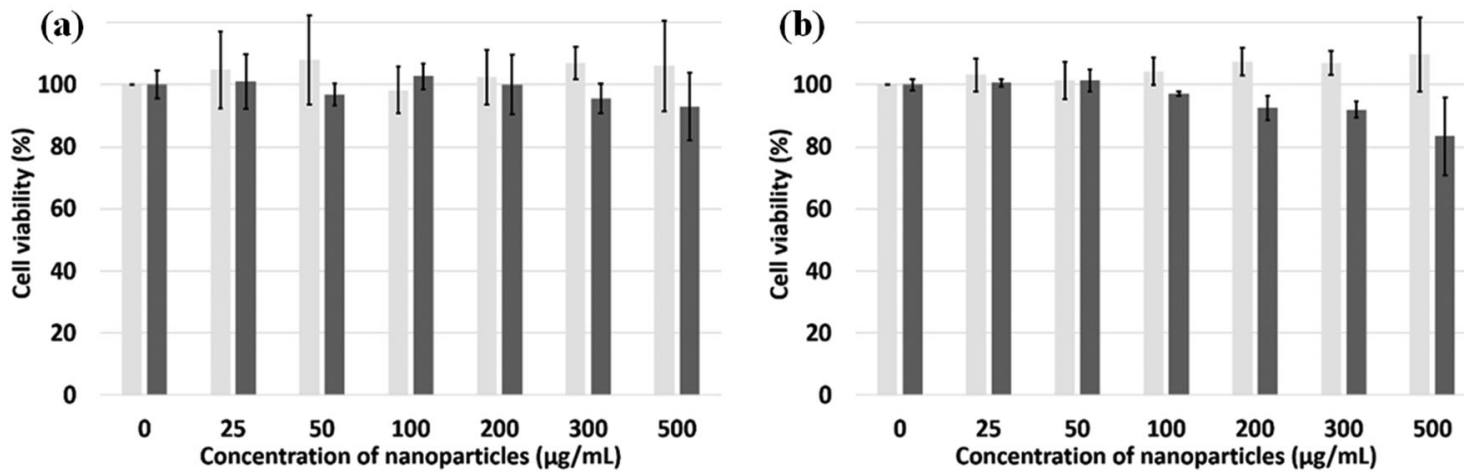

Fig. 5 Cell viability using CCK-8 assay (light grey, $n=6$ ) and the trypan blue test (dark grey, $n=3$ ). (a) Hek293 and (b) NIH3T3 cells incubated with silica NPs for 24 hours.
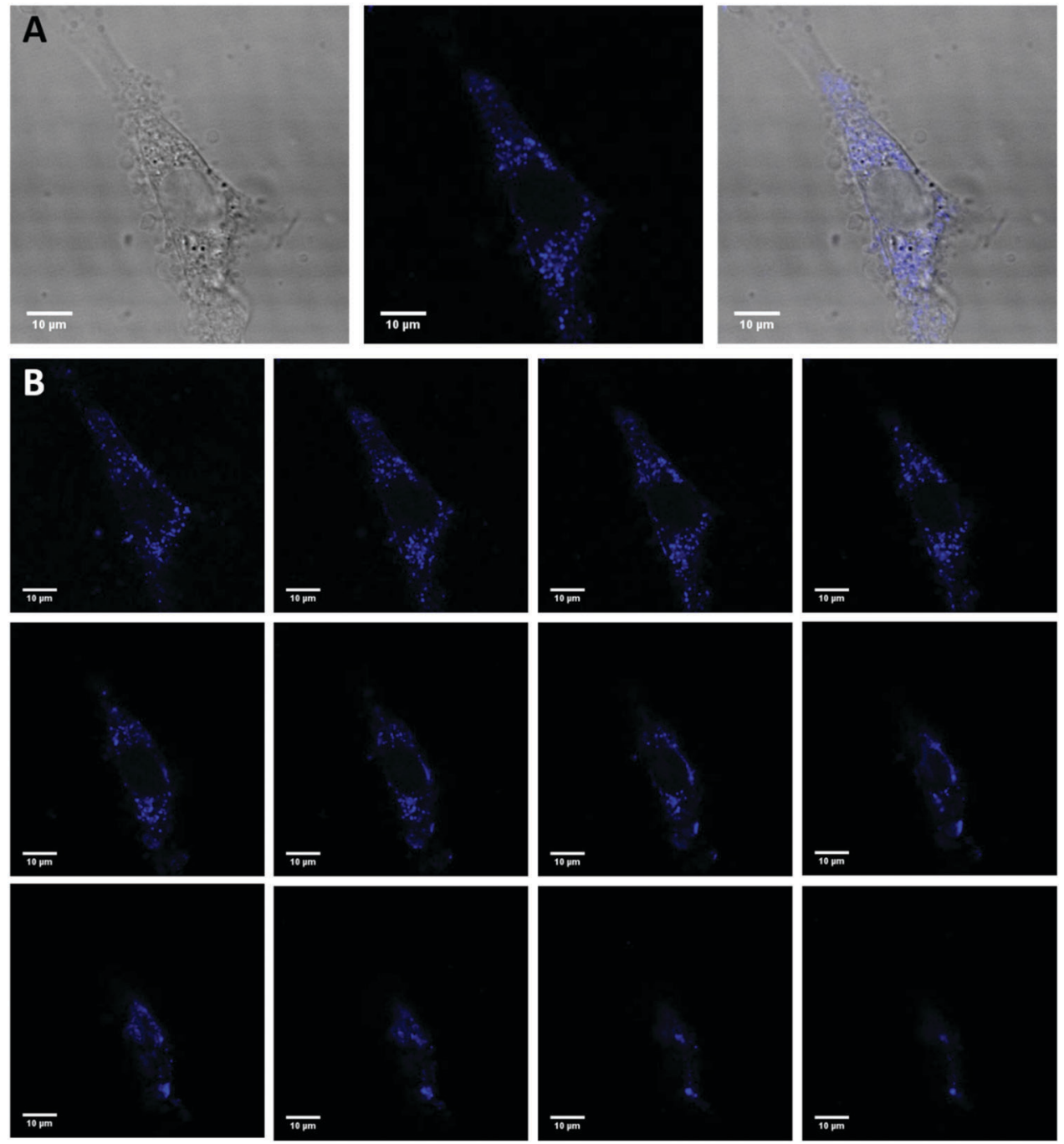

Fig. 6 Confocal microscopy of NIH3T3 cells incubated with silica NP for $24 \mathrm{~h}$ in serum containing media (DMEM $+10 \%$ FBS) and then fixed with paraformaldehyde. (A) Differential interference contrast (DIC) image (left), confocal fluorescence image (middle) and overlapped (right) image of a cell representative of the experiment. (B) Individual Z-stacks (1.0 $\mu \mathrm{m}$ intervals) of the same cell are shown.

They are taken up by cells where they are readily detected by their bright blue luminescence upon excitation at $405 \mathrm{~nm}$. The NPs should find applications in various fields in view of the ease of their preparation, their outstanding colloidal and photochemical stability, and their high PL QY. 


\section{Acknowledgements}

This study was supported by the WPI-Program and NIMS Molecule \& Material Synthesis Platform in "Nanotechnology Platform Project" both operated by the Ministry of Education, Culture, Sports, Science and Technology (MEXT), Japan. NS would like to thank Kakenhi (No. 26390024) of MEXT, and the Sumitomo Foundation.

\section{References}

1 X. He, H. Nie, K. Wang, W. Tan, X. Wu and P. Zhang, Anal. Chem., 2008, 80, 9597-9603.

2 J. L. Vivero-Escoto, R. C. Huxford-Phillips and W. Lin, Chem. Soc. Rev., 2012, 41, 2673-2685.

3 H. Ow, D. R. Larson, M. Srivastava, B. A. Baird, W. W. Webb and U. Wiesner, Nano Lett., 2005, 5, 113-117.

4 H. M. Liu, S. H. Wu, C. W. Lu, M. Yao, J. K. Hsiao, Y. Hung, Y. S. Lin, C. Y. Mou, C. S. Yang, D. M. Huang and Y. C. Chen, Small, 2008, 4, 619-626.

5 K. M. L. Taylor-Pashow, J. Della Rocca, R. C. Huxford and W. Lin, Chem. Commun., 2010, 46, 5832-5849.

6 W. Stober, A. Fink and E. Bohn, J. Colloid Interface Sci., 1968, 26, 62-69.

7 Y. Chan, J. P. Zimmer, M. Stroh, J. S. Steckel, R. K. Jain and M. G. Bawendi, Adv. Mater., 2004, 16, 2092-2097.

8 R. P. Bagwe, C. Yang, L. R. Hilliard and W. Tan, Langmuir, 2004, 20, 8336-8342.

9 A. Van Blaaderen and A. Vrij, Langmuir, 1992, 8, 2921-2931.

10 X.-H. Wang, A. R. Morales, T. Urakami, L.-F. Zhang, M. V. Bondar, M. Komatsu and K. D. Belfield, Bioconjugate Chem., 2011, 22, 1438-1450.

11 L. Xue, B. Li, Q. Fei, G. Feng, Y. Huan and Z. Shi, Nanotechnology, 2010, 21, 215502.

12 A. Burns, H. Owand and U. Weisner, Chem. Soc. Rev., 2006, 35, 1028-1042.

13 L. D. Carlos, R. A. S. Ferreira, V. de Zea Bermudez and S. J. L. Ribeiro, Adv. Mater., 2009, 21, 509-534.

14 B. Prasad, N. Nikolskaya, D. Connolly, T. Smith, S. J. Byrne, V. Gerard, Y. Gun'ko and Y. Rochev, J. Nanobiotechnol., 2010, 8, 1.

15 A. M. Jakob and T. A. Schmedake, Chem. Mater., 2006, 18, 3173-3175.

16 C. D. S. Brites, V. T. Freitas, R. A. S. Ferreira, A. Millan, F. Palacio and L. D. Carlos, Langmuir, 2012, 28, 8190-8196.

17 G. L. Davies, J. E. McCarthy, A. Rakovich and Y. K. Gunko, J. Mater. Chem., 2012, 22, 7358-7365.

18 F. Mahtab, Y. Yu, J. W. Y. Lam, J. Liu, B. Zhang, P. Lu, X. Zhang and B. Z. Tang, Adv. Funct. Mater., 2011, 21, 1733-1740.
19 N. H. N. Kamarudin, A. A. Jalil, S. Triwahyono, N. F. M. Salleh, A. H. Karim, R. R. Mukti, B. H. Hameed and A. Ahmad, Microporous Mesoporous Mater., 2013, 180, 235-241.

20 V. R. Rai and S. Agarwal, Chem. Mater., 2011, 23, 2312-2316.

21 R. M. Pasternack, S. R. Amy and Y. J. Chabal, Langmuir, 2008, 24, 12963-12971.

22 (a) B. Ghosh, Y. Masuda, Y. Wakayama, Y. Imanaka, J. Inoue, K. Hashi, K. Deguchi, H. Yamada, Y. Sakka, S. Ohki, T. Shimizu and N. Shirahata, Adv. Funct. Mater., 2014, 24, 7151-7160; (b) J. F. Moulder, W. F. Stickle, P. E. Sobel and K. D. Bomben, Handbook of X-ray photoelectron spectroscopy: a reference book of standard spectra for identification and interpretation of XPS data, Perkin-Elmer Corporation, Eden Prairie, MN, 2nd edn, 1992.

23 B. M. Gallant, X. W. Gu, D. Z. Chen, J. R. Greer and N. S. Lewis, ACS Nano, 2015, 9, 5143-5153.

24 H. Petersen, P. M. Fechner, A. L. Martin, K. Kunath, S. Stolnik, C. J. Roberts, D. Fischer, M. C. Davies and T. Kissel, Bioconjugate Chem., 2002, 13, 845-854.

25 S. Yamamoto, E. Yuba, A. Harada and K. Kono, Langmuir, 2015, 31, 8583-8588.

26 A. Paul and A. Samanta, J. Chem. Sci., 2006, 118, 335-340.

27 Y. Yang, J. Cui, M. Zheng, C. Hu, S. Tan, Y. Xiao, Q. Yang and Y. Liu, Chem. Commun., 2012, 48, 380.

28 D. S. English, L. E. Pell, Z. Yu, P. F. Barbara and B. A. Korgel, Nano Lett., 2002, 2, 681-685.

29 R. Xie and X. Peng, J. Am. Chem. Soc., 2009, 131, 10645-10651.

30 B. Ghosh, M. Takeguchi, J. Nakamura, Y. Nemoto, T. Hamaoka, S. Chandra and N. Shirahata, Sci. Rep., 2016, 6, 36951.

31 P. Yu, X. Wen, Y. R. Toh and J. Tang, J. Phys. Chem. C, 2012, 116, 6567-6571.

32 H. S. Kwack, Y. Sun and Y. H. Cho, Appl. Phys. Lett., 2003, 83, 2901-2903.

33 K. S. Min, K. V. Shcheglov, C. M. Yang, H. A. Atwater, M. L. Brongersman and A. Polman, Appl. Phys. Lett., 1996, 69, 2033.

34 T. Brankova, V. Bekiari and P. Lianos, Chem. Mater., 2003, 15, 1855.

35 N. M. Park, T. S. Kim and S. J. Park, Appl. Phys. Lett., 2001, 78, 2575.

36 M. J. Estes and G. Moddel, Phys. Rev. B: Condens. Matter Mater. Phys., 1996, 54, 14633-14642.

37 A. Puzder, A. J. Williamson, J. C. Grossman and G. Galli, J. Chem. Phys., 2002, 117, 6721-6729.

38 S. Chandra, B. Ghosh, G. Beaune, U. Nagarajan, T. Yasui, J. Nakamura, T. Tsuruoka, Y. Baba, N. Shirahata and F. M. Winnik, Nanoscale, 2016, 8, 9009-9019. 